\title{
Dysregulation of type I interferon responses in COVID-19
}

Dhiraj Acharya, GuanQun Liu and Michaela U. Gack $\mathbb{D}^{凶}$

Infection with SARS-CoV-2 can lead to excessive production of pro-inflammatory cytokines, but the production of type I interferons, which are key antiviral mediators, is reportedly blunted. Here, we discuss how imbalanced interferon responses may contribute to the pathology of COVID-19.

COVID-19 is characterized by a mild to severe respiratory illness that appears to be influenced by age and comorbidities. Critically ill patients often develop acute respiratory distress syndrome (ARDS) or multi-organ injuries as a result of secondary haemophagocytic lymphohistiocytosis (sHLH). Both ARDS and sHLH are characterized by overzealous cytokine production and excessive inflammation, the hallmarks of cytokine release syndrome (CRS). The CRS elicited by SARS-CoV-2 is reminiscent of the immune dysregulation caused by other highly pathogenic respiratory viruses, including the related betacoronaviruses SARS-CoV and MERS-CoV. Markedly elevated plasma levels of pro-inflammatory cytokines, including IL-6 and tumour necrosis factor (TNF), as well as of several chemokines have been detected in patients with COVID-19 (REF. ${ }^{1}$ ).

Airborne SARS-CoV-2 infections in humans initiate from the virus entering nasal and airway epithelial cells through binding to angiotensin-converting enzyme 2 (ACE2). TMPRSS2, a cellular protease that activates the SARS-CoV-2 spike protein, colocalizes with ACE2 and can prime SARS-CoV-2 fusion directly at the plasma membrane. In the lungs, SARS-CoV-2 infects type I and type II alveolar epithelial cells, as well as alveolar macrophages that are among the first producers of pro-inflammatory cytokines. As key components of the immediate antiviral response, type I interferons (hereafter referred to as IFNs) are crucial for restricting viral replication and spread, through autocrine and paracrine type I IFN receptor (IFNAR) signalling. However, minimal amounts of IFNs have been detected in the peripheral blood or lungs of patients with severe COVID-19 $\left(\mathrm{REFS}^{1,2}\right)$, which is in contrast to what is seen in patients infected with highly pathogenic influenza viruses. Interestingly, although low levels of systemic IFN production appear to correlate with severe COVID-19 $\left(\right.$ REF. $\left.^{2}\right)$, the local induction of IFNs and IFN-stimulated genes (ISGs) has been noticeable in the bronchoalveolar lavage (BAL) of some critically ill patients ${ }^{3}$. This was attributed to the activation of specialized immune cells such as lung-resident dendritic cells (DCs). In particular, plasmacytoid DCs were shown to produce IFNa in response to SARS-CoV. In patients with SARS who did not receive corticosteroids, IFNa was detected in plasma during the 'pre-crisis' phase but subsided during the 'crisis' phase ${ }^{4}$. In a mouse model of SARS-CoV infection, local IFN responses in the lungs were delayed relative to peak viral replication, which impeded virus clearance and was associated with the development of CRS ${ }^{5}$. The kinetics of the systemic and the local IFN responses that occur during COVID-19 remain to be fully elucidated, as well as their respective contributions to COVID-19 pathogenesis and disease severity.

The dysregulated IFN responses are indicative of the effective immunomodulatory strategies used by betacoronaviruses. During the incubation phase, SARS-CoV-2 replicates stealthily in host cells without detectably triggering IFNs, leading to high viral loads ${ }^{1}$. Coronaviruses are known to induce the formation of membranous compartments dedicated to viral RNA synthesis and thereby conceal viral pathogen-associated molecular patterns (PAMPs; for example, viral RNAs) from detection by host pattern recognition receptors (PRRs), such as RIG-I and MDA5. Furthermore, several conserved betacoronavirus proteins, predominantly non-structural proteins (nsps), are known to exert direct IFN-antagonistic activities. Some modify specific features of the viral RNA (by catalysing guanosine-N7 and ribose-2'-O methylation) to avoid recognition by specific PRRs (for example, nsp14 and nsp16), while others, such as nsp3 and nsp1, inhibit the signal transduction mediated by PRRs and by IFNAR, respectively ${ }^{5}$. By contrast, the nucleocapsid protein of SARS-CoV has been shown to directly activate NF- $\kappa B$. The robust production of pro-inflammatory cytokines and chemokines, with a limited production of IFNs, during SARS-CoV-2 infection suggests effective activation of NF- $\mathrm{kB}$ but not that of IFN-regulatory factor 3 (IRF3) and IRF7 (REF. ${ }^{1}$ ). It will be important to determine exactly how SARS-CoV-2 antagonizes IFN induction and IFNAR signalling.

As a central liaison between the innate and adaptive immune systems, IFNs are imperative to regulating the activation and functions of various immune cell populations. Importantly, during SARS-CoV or 
MERS-CoV infection in mice, IFNs directly regulate the pulmonary infiltration of monocyte-derived macrophages. Whereas blocking IFNAR signalling markedly reduced macrophage infiltration, delayed IFN induction by SARS-CoV led to the accumulation of highly activated macrophages in the lungs that induced immunopathology $y^{5}$. By contrast, IFNAR inhibition enhanced the recruitment of neutrophils to the lungs in MERS-CoV-infected mice, leading to elevated production of pro-inflammatory cytokines ${ }^{6}$. Impaired IFN production during severe COVID-19 may also lead to an imbalance in the pro-inflammatory versus pro-repair functions of airway macrophages. Patients who died from SARS-CoV showed an accumulation of pro-inflammatory macrophages but a deficiency in wound-healing macrophages in the lungs; this was associated with higher serum levels of neutralizing antibodies against the spike protein of SARS- $\mathrm{CoV}^{7}$. Other innate immune cells such as natural killer (NK) cells are also regulated by IFNs during coronavirus infection. Inhibition of IFNAR signalling suppressed the accumulation of NK cells in the lungs of MERS-CoV-infected mice $^{6}$, which may dampen the early clearance of virusinfected cells. While patients with severe COVID-19 showed profound depletion and functional exhaustion of NK cells ${ }^{8}$, it is unclear whether this NK cell dysfunction is due to dysregulation of IFN responses.

Severe COVID-19 is associated with impaired T cell responses that manifest as lymphopenia and functional exhaustion of $\mathrm{CD}^{+}$and $\mathrm{CD} 8^{+} \mathrm{T}$ cells ${ }^{8}$. Impaired $\mathrm{T}$ cell responses can result from deficient IFN production, as IFNs promote the survival and effector functions of $\mathrm{T}$ cells. Blocking IFNAR signalling during MERS-CoV infection attenuated the development of virus-specific $\mathrm{CD}^{+}$and $\mathrm{CD}^{+} \mathrm{T}$ cells in mice ${ }^{6}$. Although the early production of IFNs is crucial for an efficient $\mathrm{T}$ cell response, a delayed IFN response can inhibit $\mathrm{T}$ cell proliferation or T cell egress from lymphoid organs, or it can cause functional exhaustion and cell death of T cells. The lung injury associated with CRS in patients with severe COVID-19 indicates a possible failure to activate immunosuppressive mechanisms in a timely manner. Indeed, regulatory $\mathrm{T}\left(\mathrm{T}_{\text {reg }}\right)$ cell counts in patients with COVID-19 have been shown to inversely correlate with disease severity ${ }^{9}$. IFNs are known to be crucial regulators of the development of $\mathrm{T}_{\text {reg }}$ cells. It is thus tempting to speculate that the deficient or dysregulated IFN responses elicited by SARS-CoV-2 infection may influence the generation of $\mathrm{T}_{\text {reg }}$ cells during the recovery phase of COVID- 19. Future studies should explore how IFN dysregulation during COVID-19 might shape T cell responses and, given that $\mathrm{CD}^{+} \mathrm{T}$ cell activation is crucial for the development of B cell immunity, how this may in turn affect antibody responses.

That IFN dysregulation represents a key determinant of COVID-19 pathogenesis highlights its potential for therapeutic intervention. Prophylactic administration of IFNs, which elicits a pre-existing antiviral state in target cells, may block viral infection at the very early stage. Daily IFNa nasal drops along with standard personal protective equipment (PPE) were shown to protect at-risk health-care workers from COVID-19 over 28 days without noticeable adverse effects (NCT04320238). The use of IFNs as a treatment for COVID-19 remains controversial, particularly regarding the timing of administration. Early IFN treatment before peak viral replication protected mice from lethal SARS-CoV or MERS-CoV challenge, whereas late IFN administration impeded viral clearance and aggravated immunopathology $^{5,6}$. Clinical studies on SARS-CoV and MERS-CoV have also shown inconclusive effects of IFN in combination with antivirals on disease outcomes, which is likely due to varied timing of administration and also comorbidities. Importantly, ACE2 has been recently identified as an ISG in human airway epithelial cells ${ }^{10}$. Whether prophylactic or therapeutic IFN administration may enhance the entry and replication of SARS-CoV-2 in target cells during disease progression is a potential safety concern. Further studies should also determine the contributions of host genetics, age and comorbidities to the therapeutic effectiveness of IFNs. While several ongoing clinical trials are evaluating the efficacy of IFN treatment for COVID-19, a deeper understanding of the spatiotemporal kinetics of IFN responses during clinical SARS-CoV-2 infections is warranted to inform IFN-related therapeutics and vaccine design.

1. Blanco-Melo, D. et al. Imbalanced host response to SARS-CoV-2 drives development of COVID-19. Cell https://doi.org/10.1016/j. cell.2020.04.026 (2020)

2. Hadjadj, J. et al. Impaired type I interferon activity and exacerbated inflammatory responses in severe Covid-19 patients. Preprint at medRxiv https://doi.org/10.1101/2020.04.19.20068015 (2020).

3. Zhou, Z. et al. Heightened innate immune responses in the respiratory tract of COVID-19 patients. Cell Host Microbe https://doi.org/10.1016/j.chom.2020.04.017 (2020).

4. Cameron, M. J. et al. Interferon-mediated immunopathological events are associated with atypical innate and adaptive immune responses in patients with severe acute respiratory syndrome. J. Virol. 81, 8692-8706 (2007).

5. Channappanavar, R. et al. Dysregulated type I interferon and inflammatory monocyte-macrophage responses cause lethal pneumonia in SARS-CoV-infected mice. Cell Host Microbe 19, 181-193 (2016).

6. Channappanavar, R. et al. IFN-I response timing relative to virus replication determines MERS coronavirus infection outcomes. J. Clin. Invest. 130, 3625-3639 (2019).

7. Liu, L. et al. Anti-spike IgG causes severe acute lung injury by skewing macrophage responses during acute SARS-CoV infection. JCI Insight 4, e 123158 (2019).

8. Zheng, M. et al. Functional exhaustion of antiviral lymphocytes in COVID-19 patients. Cell Mol. Immunol. 17, 533-535 (2020).

9. Qin, C. et al. Dysregulation of immune response in patients with coronavirus 2019 (COVID-19) in Wuhan, China. Clin. Infect. Dis. https://doi.org/10.1093/cid/ciaa248 (2020).

10. Ziegler, C. G. K. et al. SARS-CoV-2 receptor ACE2 is an interferonstimulated gene in human airway epithelial cells and is detected in specific cell subsets across tissues. Cell https://doi.org/10.1016/ j.cell.2020.04.035 (2020).

Author contributions

The authors contributed equally to all aspects of the article.

Competing interests

The authors declare no competing interests.

RELATED LINKS

ClinicalTrials.gov: https://clinicaltrials.gov/ 